\title{
Diagnostics for laser plasma EUV sources
}

\author{
M. Richardson, ${ }^{\text {a) }}$ C-S. Koay, K. Takenoshita,

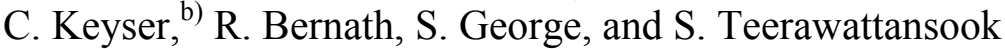 \\ Laser Plasma Laboratory, College of Optics and Photonics: CREOL \& FPCE \\ University of Central Florida, Orlando, Florida
}

\begin{abstract}
A high repetition-rate laser plasma source, possessing distinct radiation and particle emission characteristics, is now a principal candidate light source for the next generation of technology for the fabrication of computer chips. For these sources to satisfy this critical need they will need to meet unprecedented levels of performance, stability and lifetime. We review here some of the principal diagnostics of the EUV radiation that are now being utilized in the metrology, spectroscopy and imaging of these sources.
\end{abstract}

Keywords: laser plasma, EUV diagnostics, EUV sources

\section{INTRODUCTION}

EUV Lithography (EUVL) is currently undergoing intense research and development worldwide in a concerted international effort. It is now considered as the most viable technology to overtake optical lithography in about 2009 for high-volume production of computer chips of feature sizes at and below the $32 \mathrm{~nm}$ node. The need for this transition in technology, from Deep UV lithography to EUVL, probably the largest technology change-out in the history of the computer industry, is a consequence of the ever-decreasing size requirement of minimum feature size in computer chip circuits, commonly attributed to Moore's Law. This minimum size is governed by the diffraction of the light that is used to expose or print in the lithographic process. This depends on the wavelength of the light source, as expressed in

$$
W=k_{1} \frac{\lambda}{N A}
$$

where $\lambda$ is the wavelength of the light source, $k_{l}$ is a constant related to the imaging scheme, and $N A$ is the numerical aperture of the optical imaging system exposing the resist. In current UV lithography systems with $248 \mathrm{~nm}$ or $193 \mathrm{~nm}$ excimer laser sources, the minimum feature size $\mathrm{W}$ has been reduced progressively with the use of various resolution enhancement techniques ${ }^{1}$ and by manipulating $\mathrm{k}_{1}$ and NA. With $\mathrm{k}_{1}$ values now being pressed below 0.5 and the complex design of expensive multi-element UV exposure optics possessing NA values close to 0.8 , there is little room left in this technology for further improvements in $\mathrm{W}$. The only remaining parameter to change is the wavelength, thus the reason for the transition from optical wavelength to EUV light with wavelength of $13.5 \mathrm{~nm}$.

The need for a high power EUV light source remains at the top of the list among other challenges that need to be overcome to make EUV lithography successful. Both laser plasma sources and discharge plasma sources are being developed to meet this need: a stable, debris-free light source that provides useful EUV radiation, which lies within a $2 \%$ spectral bandwidth at $13.5 \mathrm{~nm}$, with power levels $>115 \mathrm{~W}$ at an intermediate focus ${ }^{2}$. The latter implies powers emitted into $2 \pi$ steradians at the source itself in excess of $200 \mathrm{~W}$.

Laser-produced plasmas, in principle, have many attractive features as sources for EUVL. Since the laser energy is delivered to the source by optical coupling, the laser and its associated infrastructure can be located separate from the stepper, unlike a discharge source, which requires much of its cooling and power system to be integral. Depending on geometry and laser architecture, a laser plasma source can easily extend to greater dose stability because the source's repetition rate can extend conveniently beyond $10 \mathrm{kHz}$. Moreover, laser plasmas have a wide margin on the source's size

\footnotetext{
a) Email: mcr@creol.ucf.edu

b) Present address: Division of Space Sciences, Naval Research Laboratories, Washington DC
} 
requirement. Because all EUV steppers are expected to have an etendue in the range of $1-3.3 \mathrm{~mm}^{2} \cdot \mathrm{sr}$, this implies a limit for the source size of $<1 \mathrm{~mm}$. The sizes of discharges sources are likely to exceed this limit as the power levels are pushed up to meet the power requirement. Since laser plasma source size is considerably smaller than discharge sources, it can achieve higher power operation through increased repetition-rates.

For a laser plasma source to be successful, it must meet very high long-term performance levels, that is, it is required to operate continually for periods of $\sim 1$ year at repetition rate of $7-10 \mathrm{kHz}$, or greater, with a pulse-to-pulse energy stability $<2 \%$. In addition, the source must work in a scheme that can prevent the large numerical aperture $(>0.25)$ collection optics in the lithography stepper from suffering the deleterious effects of target debris. Apart from the strict condition of debris-free operation of the source, the conversion efficiency (CE) of the source into the useful EUV energy becomes an important parameter to be optimized. For a laser plasma source, the $\mathrm{CE}$ must be sufficiently large to (i) provide the projected required collectable power levels with viable commercial lasers and (ii) permit the overall cost of the source, including the laser, to remain within economic models of the overall EUV lithography stepper tool.

From the outset of our involvement in laser plasma sources for lithography, we have expressed concern for the effects of material debris from the target on the collection optics. Early investigations of tin as a source material, by others and ourselves $^{3,4}$, showed its potential as an efficient emitter at $13 \mathrm{~nm}$, but simple witness plate tests identified high velocity, micron-size aerosols flying from the target ${ }^{5}$ with high velocities, convinced us and others of the danger of using solid metallic targets. Our approach has since been to configure the laser plasma from a mass-limited target, i.e. one whose mass only equals the total number of atomic radiators required. With this target concept, for example of a $100 \mathrm{~mJ}$ laser pulse (energy $\sim 6 \times 10^{18} \mathrm{eV}$ ) will produce $\mathrm{a} \sim 30 \mathrm{eV}$ plasma of $\sim 10^{15}$ atoms, taking into account the energy needed for ionization and excitation. Depending on the material, such a target will have a diameter of $\sim 10 \mu \mathrm{m}$. Assuming that this target is uniformly energized, there should be no particulate debris all. The sole problem will now be to protect the collection mirrors from the ions coming from the plasma. Ions, as opposed to particulate neutral matter, can be controlled by electric and magnetic fields, and are slowed down by recombination. With this philosophy we began investigating the water droplet target, which, serendipitously emits a strong narrow line at $13 \mathrm{~nm}$ (the $4 \mathrm{~d}-2 \mathrm{p}$ transition in Li-like Oxygen) ${ }^{4,6}$. Another approach which quickly gained favor was to use jets of liquid Xe atomic clusters, produced by pulsed supersonic ejection through a microscopic nozzle ${ }^{7,8}$. Xenon was recognized as a broadband emitter of EUV radiation from previous generalized studies of soft-X-ray emission from laser plasmas. It was felt that Xe, being a gas would pose less serious threats to collection mirrors. Subsequently liquid jet ${ }^{10}$ and liquid Xe droplet ${ }^{11}$ targets have been developed. More recently, we have introduced the tin-doped liquid droplet target, which combines the advantages of tin with the mass-limited target concept ${ }^{12}$.

Our group has parallel programs in theory and experiments to develop an efficient laser plasma EUV source. To attain a comprehensive understanding of the plasma dynamics as well as spectral emission, theoretical simulations and optical diagnostics of the laser plasma are being made. In this paper we describe the diagnostics we use for studying plasma dynamics, source imaging, spectroscopy, and metrology.

\section{PLASMA DYNAMICS}

Interferometry is a powerful optical probe technique capable of providing high resolution spatial and temporal information about an expanding plasma. We have employed the polarized light interferometer to study the water droplet plasma expansion dynamics ${ }^{13,14}$. Figure 1 illustrates the simplicity of this device. The lens L images the object $\mathrm{O}$, the plasma, into the image plane. The Wollaston prism creates two orthogonally polarized images that overlap in the image plane and are separated by an angle $\varepsilon$. Interference between these two images is accomplished by a pair of polarizers, $\mathrm{P}_{1}$ and $\mathrm{P}_{2}$, oriented either parallel or perpendicular to one another. In order to form high contrast fringes in the image plane, $\mathrm{P}_{2}$ is oriented such that the two originally orthogonal images will, after passing through $\mathrm{P}_{2}$, have the same polarization and intensity. The fringe separation in the object plane is given by $i=(\lambda f / b \varepsilon)$, where $\lambda$ is the wavelength, $f$ is the focal length of the imaging lens, $\varepsilon$ is the angle between the two deviated images, and $b$ is the distance between the Wollaston prism and the lens focal plane ${ }^{13}$. The fringe orientation can be changed by rotating the polarization elements and the fringe spacing is varied by changing the distance $b$. The Wollaston prism used in our experiments is composed of Quartz and has $\varepsilon=8.7 \mathrm{mrad}$. Using a $7.5 \mathrm{~cm}$ focal length lens and $b=37.7 \mathrm{~cm}$ the fringe spacing is calculated to be $12.1 \mu \mathrm{m}$. 


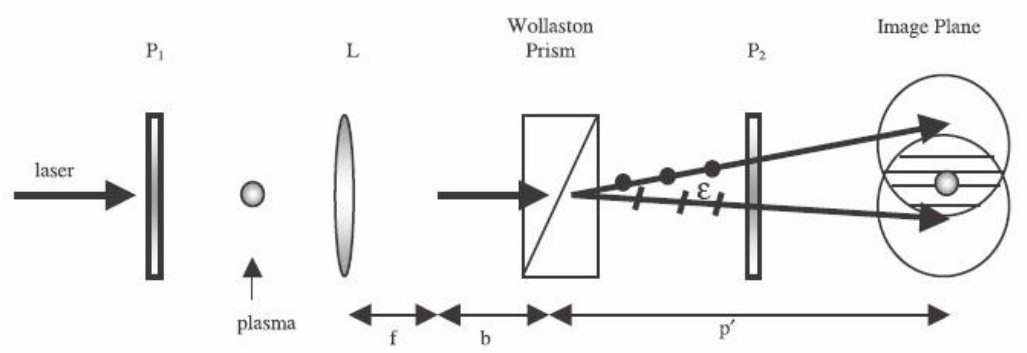

Figure 1: Polarized light interferometer

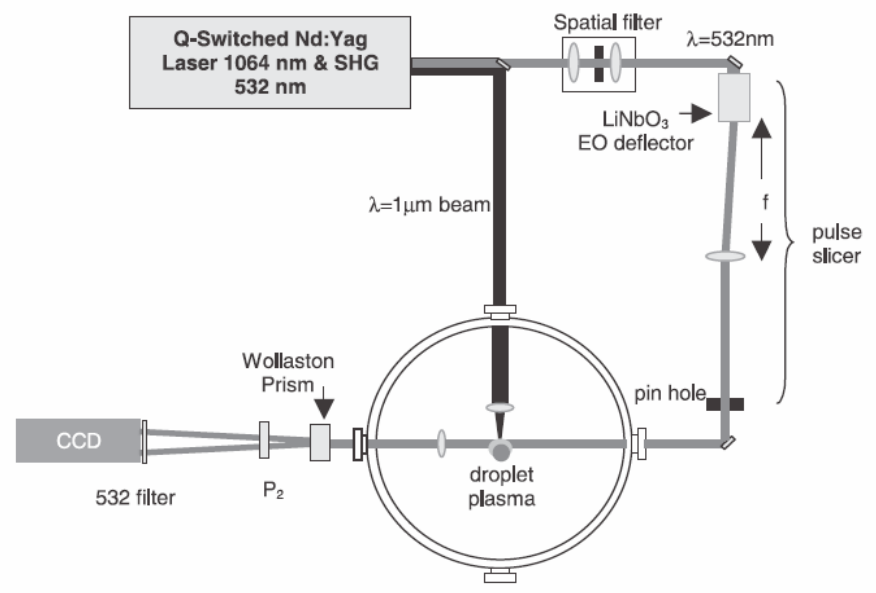

Figure 2: Experimental set up for interferometry of the laser plasma

The cumulative phase difference between light rays passing through equal lengths of plasma and vacuum is given by ${ }^{15}$

$\Delta \phi=k_{0} \int\left(\left(1-\frac{n_{e}}{n_{c}}\right)^{1 / 2}-1\right) d l \approx \frac{k_{0}}{2 n_{c}} \int n_{e} d l$

where the plasma index of refraction is $N=\left(1-n_{e} / n_{c}\right)^{1 / 2}, n_{e}$ is the electron density, and $n_{c}=m_{e} \varepsilon_{o} \omega^{2} / 4 \pi e^{2}$ is the critical density. In writing the expression on the right side it was assumed that $n_{e}<<n_{c}$. In the event that the plasma is spherically or cylindrically symmetric one can extract the radial distribution of the electron distribution from the above chordal integral by applying the Abel inversion given by ${ }^{15}$

$n_{e}(r)=\frac{n_{c}-1}{k_{0} \pi} \int_{r}^{R} \frac{d \Delta \phi}{d y} \frac{1}{\sqrt{y^{2}-r^{2}}} d y$

Here $k_{0}$ is the probe wave number, $R$ is the plasma radius where $n_{\mathrm{e}}=0$, and $y$ is the coordinate transverse to the axis of symmetry and the line of sight. The code Interferometric Data Evaluation Algorithms (IDEA) was used to calculate the plasma induced phase shift and compute Abel inversion ${ }^{16}$. Figure 2 illustrates the experimental set up being used for the optical diagnostic experiments. The ionizing laser beam is produced by a Spectra Physics $Q$-switched Nd:YAG laser operating at $100 \mathrm{~Hz}$, having $10 \mathrm{~ns}(\mathrm{FWHM}), 270 \mathrm{~mJ}$ pulses that are focused to $4 \times 10^{11} \mathrm{~W} / \mathrm{cm}^{2}$ on target by a $\mathrm{f} / 2$ lens. Due to the rapid expansion rates of laser plasmas a short probe pulse is necessary to avoid fringe blurring in the interferograms. The probe beam is initially formed by passing the Nd:YAG fundamental beam through a second harmonic generator (SHG) producing a $6 \mathrm{~ns}, 532 \mathrm{~nm}$ beam. Following the SHG, the $532 \mathrm{~nm}$ beam is spatially filtered to remove beam non-uniformities. The probe pulse is further shortened to a duration of about $1 \mathrm{~ns}$ by the application of a pulse slicing technique ${ }^{17}$. A $5 \mathrm{kV}, 10 \mathrm{~ns}$ rise time voltage pulse is applied to a $\mathrm{LiNbO}_{3}$ crystal having a quadrupole 
electrode arrangement as the SHG pulse passes through. The high voltage pulse produces a time varying linear gradient in the crystal index of refraction causing an angular deflection of the pulse. By placing the exit face of the crystal in the focal plane of a $1 \mathrm{~m}$ focal length lens the deflected pulse is redirected parallel to the axis of the crystal. As this beam is swept across a $300 \mu \mathrm{m}$ pinhole, a short $1 \mathrm{~ns}$ pulse is sliced out of the original $6 \mathrm{~ns} 532 \mathrm{~nm}$ pulse. The plasma was imaged by a $f / 3$ lens with a magnification of 17.75 and a spatial resolution of about $5 \mu \mathrm{m}$. Figures 3(a) and 3(b) are interferograms showing a $35 \mu \mathrm{m}$ diameter water droplet and the plasma at the peak of the $1 \mu \mathrm{m}$ ionizing pulse, respectively; the laser is incident from the left side of the photo. The dark region in the center region of Fig. 3(b) is due to the opaqueness of the plasma above critical density $\left(n_{c}=4 \times 10^{21} \mathrm{~cm}^{-3}\right.$ for the $532 \mathrm{~nm}$ probe) and strong refraction of the probe beam by a steep electron density gradient.
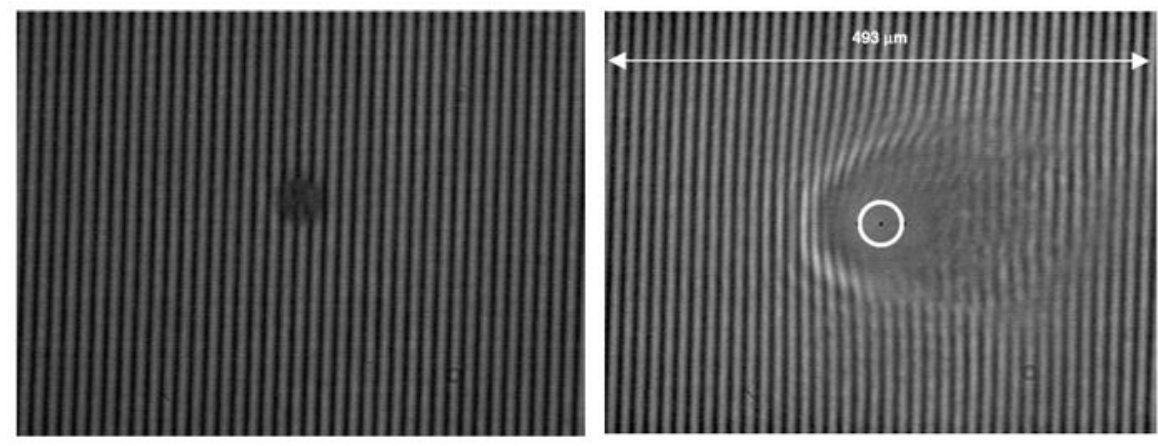

Figure 3 (a): Interferogram of a water droplet, (b) Interferogram of droplet plasma at peak of ionizing pulse

The crescent shaped region of enhanced brightness to the left of the dark region is the result of this refractive affect, which inhibits probing to the critical density. The circle in the center of the plasma indicates the approximate dimensions and position of the $35 \mu \mathrm{m}$ drop before irradiation. The circular shaped fringes in the region of direct laser droplet interaction, reveal, to first order, spherical symmetry in the expanding plasma. In the region above the drop, a plasma wake is apparent. On the rear side of the plasma there are at least two plasma jets as evidenced by a line of ripples in the fringe pattern. In the area directly behind the plasma, very small fringe shifts indicate the presence of a low density plasma. Superimposed on this quasi-uniform density pattern are perturbations in the interferogram indicating the presence of a non-uniform field of either water vapor or plasma leakage. Figure 4(a) is a plot of the Abel inverted electron density distribution along the axis of the incident laser beam. Also shown in the plot is an exponential fit to the data; the fit is extremely good indicating an exponential plasma blow off in the under-dense region of the plasma.
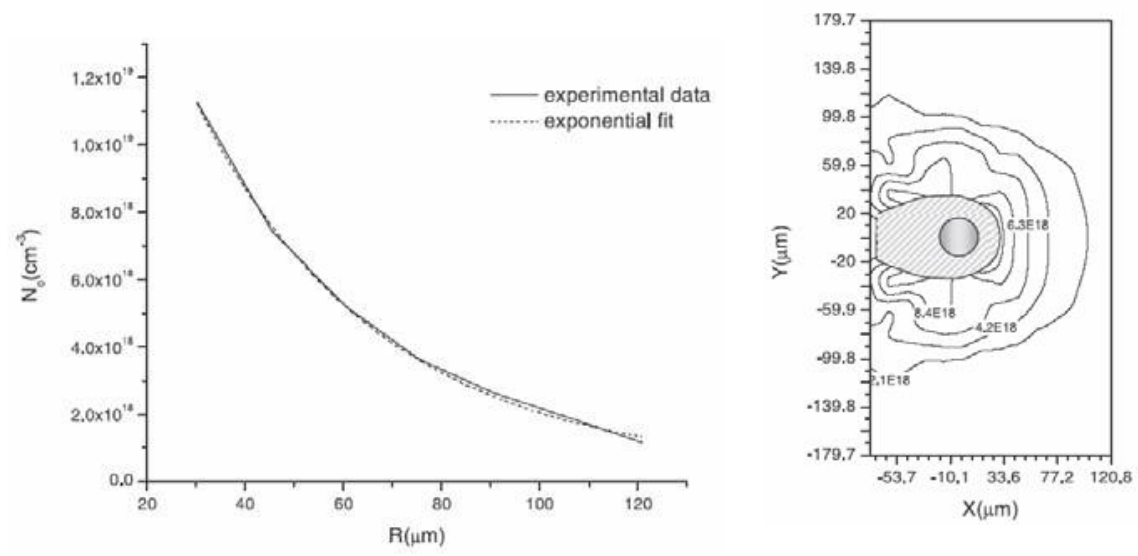

Figure 4: (a) Electron density distribution along the axis of incident laser beam, (b) Contour surface plot of electron density $\left(\mathrm{cm}^{-3}\right)$ distribution in droplet plasma 
The contour map in Fig. 4(b) displays the two dimensional results of the Abel inversion. The cross hatched region in the center of the contour plot represents the opaque area of the plasma; the circle in the cross hatched area indicates the approximate position and dimensions of the droplet before irradiation. The Abel inversion in the forward region of laser plasma interaction yields an approximately spherically symmetric electron density distribution. The contour plot also reveals the presence of a jet diagonally behind the droplet.

\section{IN-BAND SOURCE IMAGING}

We have developed a new mode of EUV radiation detection based on a $\mathrm{Ce}^{3+}$ :YAG crystal as a EUV-to-visible converter for short wavelength imaging applications. Conventional techniques rely on films, channel plates or expensive x-ray CCD's. The new technique provides an alternative to these methods. Luminescent materials have been used as radiation detectors in many applications e.g. medical imaging, tomography, and gamma-camera. We have characterized $\mathrm{Ce}^{3+}$ :YAG as an EUV-to-visible light converter, and integrated this into a novel high resolution laser-plasma, $13.5 \mathrm{~nm}$ (2\% bandwidth) imaging camera. We are also incorporating this new EUV recording technique into other devices including spectrographs.

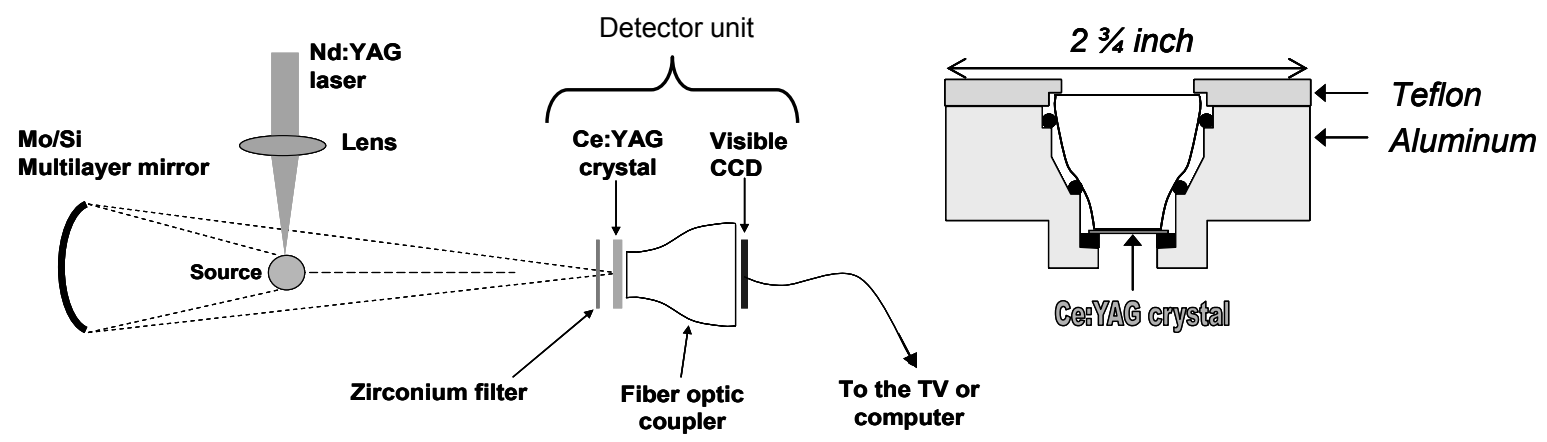

Figure 5: Experimental setup for in-band imaging and the schematic of the detector unit.

The experimental setup for the in-band source imaging is shown in Fig. 5. Wavelength selection was made by using a Mo-Si multilayer mirror and free-standing zirconium metal filter. The mirror reflects into a narrowband spectral band of $3 \%$ at $13.5 \mathrm{~nm}$, having a peak reflectance of $69 \%$ for a fresh mirror. The maximum transmission of a $0.5 \mu \mathrm{m}$ thick $\mathrm{Zr}$ filter is about $18 \%$ and passes light within 6-15 nm FWHM.

The radius of curvature of the multilayer mirror is $500 \mathrm{~mm}$ and it is used to image the source onto the $\mathrm{Ce}^{3+}: \mathrm{YAG}$ crystal (thickness of $500 \mu \mathrm{m}$ ) in the detector unit. When the EUV light irradiates the crystal, the high energy photon is absorbed by the host lattice (YAG) and is converted in lattice excitations which stimulate the transition from the lowest $5 \mathrm{~d}$ state to the $4 \mathrm{f}$ state (ground state) of the $\mathrm{Ce}^{3+}$. This transition is accompanied by the emission of photons at $550 \mathrm{~nm}$ in the visible spectrum $^{18}$. A fiber optic coupler is used to relay the visible light image from the crystal to a generic visible CCD camera.

The imaging system's magnification can be adjusted according to the usual imaging equation of a spherical mirror system. The fiber output coupler, in its taper form, provides a magnification of 2.25 . With a magnification of 5 in the optical system, the total magnification of the whole camera is $2.25 \times 5 \approx 11$. Figure 6 (a) shows an image obtained by using this camera. For comparison, an image of the same source obtained with the Flying Circus 2 imager ${ }^{19}$, which has a total magnification of 2 and employed a back-thinned x-ray CCD camera, is shown in Fig. 6(b). Both techniques give the same result for measurement of the source size, though the latter suffered from pixelation due to small magnification. 

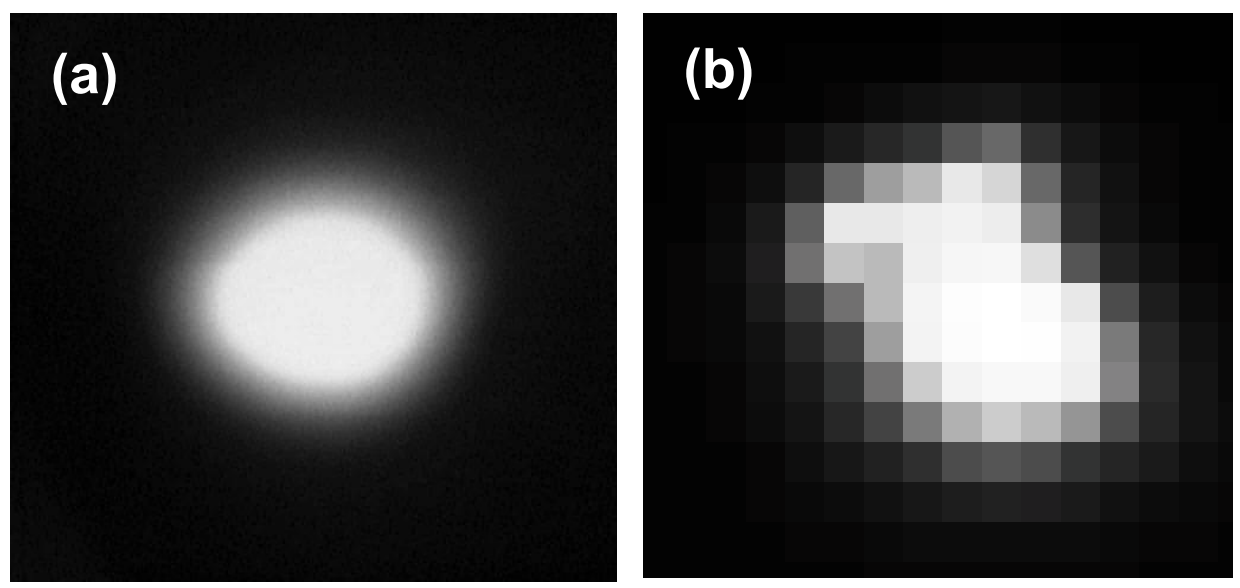

Figure 6: Inband source image showing a source size of $\sim 75 \mu \mathrm{m}$ diameter obtained with (a) the new Ce $\mathrm{BAG}^{3+}$ :YAstal imager and (b) FC2 imager.

\section{SPECTROSCOPY}

Soft x-ray spectroscopy is a powerful tool for determining characteristics of the laser plasma source's emission responsible for the highest production of useful EUV light. In our studies we use two types of spectrometers, a transmission grating spectrograph (TGS) ${ }^{20}$ and a so-called, "flat-field' spectrograph (FFS) which uses a variable-spaced (Harada) grazing incidence grating. Both instruments were developed specifically for these EUV studies. Details of these instruments can be found elsewhere.

The TGS employs a free standing 5000 line/mm (200nm period) grating from XOPT. ${ }^{21}$ The grating bars are made of gold and are $0.5 \mu \mathrm{m}$ thick. Due to the grating's submicron period, a wire mesh is used to provide mechanical support for the grating. The wire mesh is composed of coarse wires having $30 \mu \mathrm{m}$ wide bars with an effective period of $150 \mu \mathrm{m}$ and crossed fine wires having $1.7 \mu \mathrm{m}$ thick bars with an effective period of $4 \mu \mathrm{m}$. The support structure results in a $46 \%$ transmission through the grating. The diffraction efficiency of a similar XOPT 5000 line/mm freestanding grating has been measured. ${ }^{22}$

The FFS employed a Harada grating ${ }^{23}$ with a radius of curvature of $\mathrm{R}=5.649 \mathrm{~m}$, a nominal groove spacing of $\mathrm{d}_{\mathrm{o}}=1200$ lines $/ \mathrm{mm}$, and an angle of incidence of $\alpha=87^{\circ}$. The slit-grating center distance is $237 \mathrm{~mm}$, and the grating center-image plane distance is $235 \mathrm{~mm} .{ }^{4}$ The plate factor at $\lambda=10 \mathrm{~nm}$ is calculated to be $0.56 \mathrm{~nm} / \mathrm{mm}$. Using a slit width of $70 \mu \mathrm{m}$, the resolving power at $10 \mathrm{~nm}$ in first order is $\mathrm{R} \sim 880$, corresponding to a minimum resolvable wavelength of $\Delta \lambda \sim 0.01 \mathrm{~nm}$. The diffraction efficiency of a similar grating has been measured. ${ }^{24}$ A back-thinned x-ray CCD camera is used to record the dispersed spectrum ${ }^{25}$.
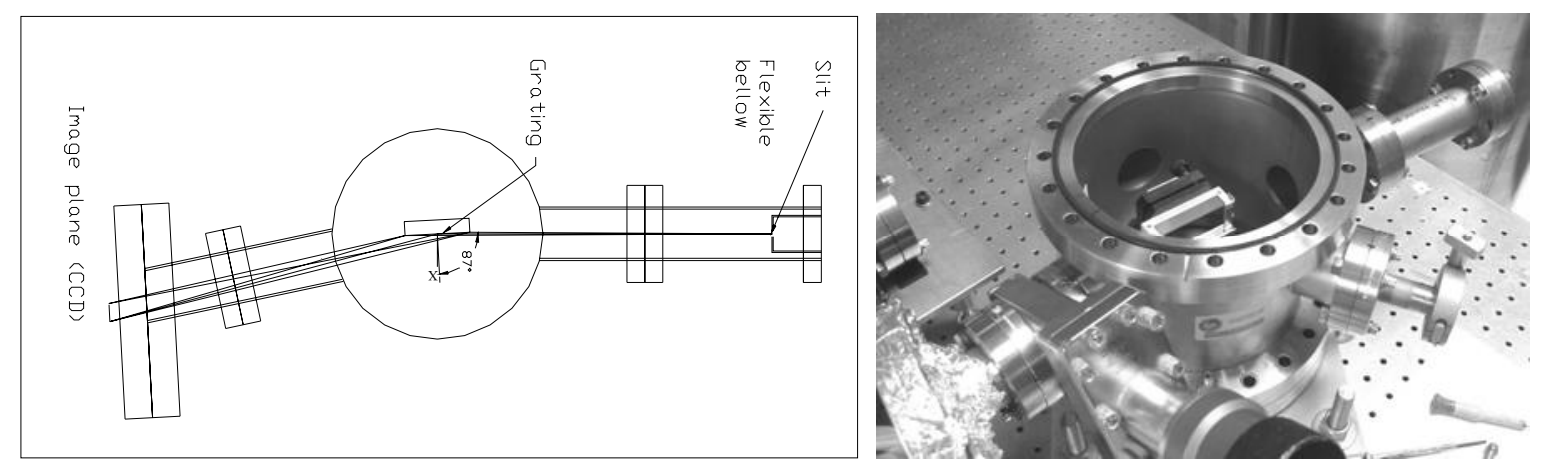

Figure 7: Schematic diagram and a photograph of the FFS. 
Spectroscopy also reveals the effects of the detailed ion structure on the unresolved transition array (UTA) emission from Sn-doped targets. Fig. 8 shows typical spectra. The broadband emission resulting from $\mathrm{Sn}^{+10}$ and other ions is observable, together with the sharp emission lines of $\mathrm{O}^{+5}$.

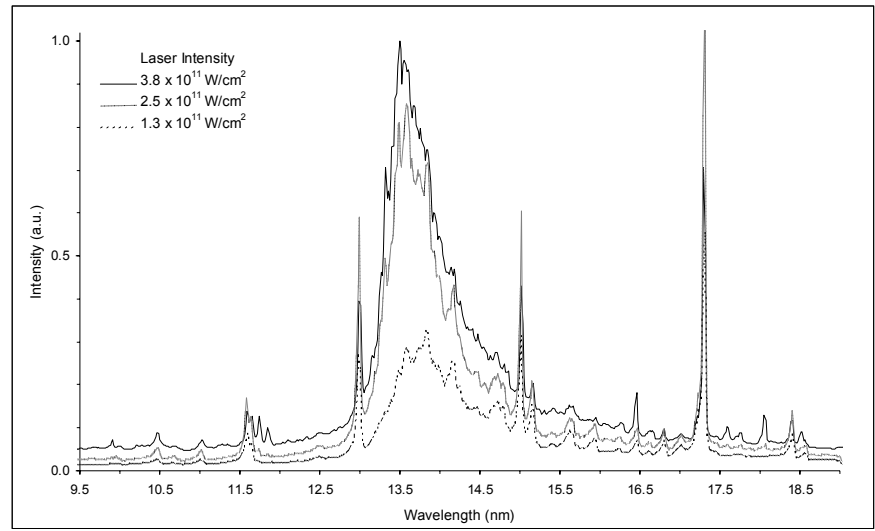

Figure 8: Spectra of tin-doped droplet targets

\section{METROLOGY}

We have adopted the same methods of EUV radiation metrology as the FOM Flying Circus (FC) group ${ }^{19}$ in estimating the effective conversion efficiency of the source. The FC is a narrowband EUV diagnostic comprising a calibrated curved normal incidence multilayer mirror and a $\mathrm{Zr}$ filtered AXUV-100 photodiode ${ }^{26}$. The Mo-Si multilayer spherical mirror collects light from the source; a fresh mirror typically has a peak reflectivity of $\sim 69 \%$ at $13.5 \mathrm{~nm}$ and a narrow pass band ( $\sim 3.7 \%$ FWHM). The photodiode has a spectral responsivity of $\sim 0.23 \mathrm{~A} / \mathrm{W}$ at $13.5 \mathrm{~nm}$, and it is operated with a reversed bias at 26 volts to ensure linearity. The transmission at $13.5 \mathrm{~nm}$ for a $0.5 \mu \mathrm{m}$ thick $\mathrm{Zr}$ metal filter is $\sim 18 \%{ }^{27}$ Calibration of the optics used in the instrument was made by NIST.

The method that we used for calculating $\mathrm{CE}$ is the same as the method employed by the $\mathrm{FC} 2$ team ${ }^{19}$. A numerical analysis program was developed for this purpose. The program takes a number of input files, uses a process of interpolation to combine these files, and finally performs numerical integrations to calculate the source's EUV energy.

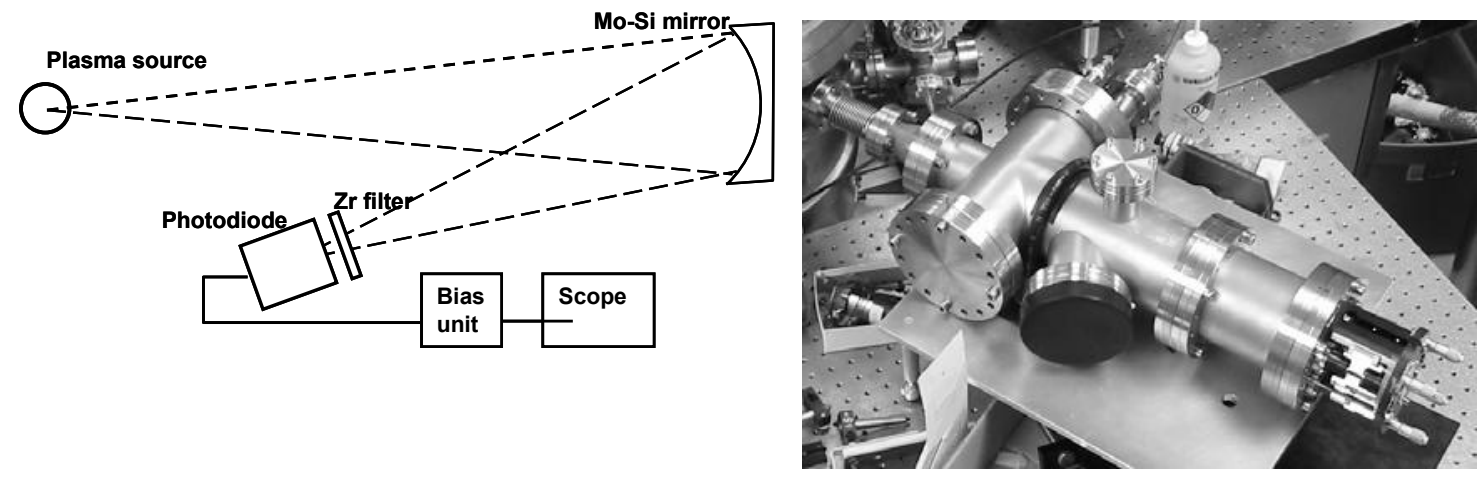

Figure 9: Schematic and photograph of the Flying Circus instrument

The EUV energy radiated by the source into a $2 \pi$ steradian solid angle and within the $2 \%$ bandwidth (centered on $13.50 \mathrm{~nm})$, is given by 


$$
E_{B W}=\frac{2 \pi A_{\text {scope }}}{\Omega R_{\text {scope }}}\left(\frac{\int_{B W} I_{s}(\lambda) d \lambda}{\int_{\text {all }} I_{s}(\lambda) T_{g}(\lambda) R_{\text {mir }}(\lambda) T_{f}(\lambda) \eta_{\text {diode }}(\lambda) d \lambda}\right)
$$

where $\Omega$ is the collection solid angle of the FC mirror subtended from the source; $\mathrm{A}_{\text {scope }}$ is the integrated area under the EUV signal waveform displayed on an oscilloscope; $R_{\text {scope }}$ is the impedance of oscilloscope channel; $T_{g}(\lambda)$ is the transmission curve of the gas in the vacuum chamber $^{27} . \mathrm{R}_{\operatorname{mir}}(\lambda)$ is the calibrated mirror reflectivity curve; $\mathrm{T}_{\mathrm{f}}(\lambda)$ is the transmission curve of filter(s) used to block visible light from entering AXUV detector; $\eta_{\text {diode }}(\lambda)$ is the calibrated responsivity curve of the AXUV detector in the $\mathrm{FC}$; and $\mathrm{I}_{\mathrm{s}}(\lambda)$ is the spectrum of the EUV source in arbitrary units.

The equation assumes isotropic emission from the source. The CE is the ratio of the EUV energy to the laser energy at target. As a convention, the $\mathrm{CE}$ is quoted in the units of "percent into $2 \pi$ steradian $\mathrm{x} 2 \% \mathrm{BW}$ at $13.5 \mathrm{~nm}$ ". In our latest result from the Sn-doped droplet target laser plasma source, a maximum conversion efficiency in excess of $2 \%$ was measured.

\section{CONCLUSION}

The development of EUV sources for lithography is demanding precision in measurement of the parameters of laser plasmas well beyond previously required studies. We have described here the methods we are currently using in the assessment of the plasma dynamics and radiation emission of micro-droplet plasmas. In the future, improvements will need to be made to the spectral resolution of spectroscopic investigations, including the spatial and temporal measurements. Moreover, although not covered here, we are using a suite of particle and ion measurement approaches to assess their energy and state ${ }^{28}$. These improvements will doubtlessly lead to improvements in desing and performance of laser-based sources for EUV lithography.

\section{ACKNOWLEDGMENTS}

The authors gratefully acknowledge the help and support of many people in this work. These include Dr. Greg Shimkaveg and Joshua Duncan from CREOL, Dr. Eric Gullickson (LBNL) for multilayer mirrors, and the FOM team (Dr. Fred Bijkerk, Santi Alonso vd Westen and Caspar Bruineman). This work is supported by contracts from SEMATECH and Intel through SRC, and by support from the State of Florida.

\section{References}

1 F. Schellenberg: Proc. SPIE, v. 5377, xix (2004)

2 Y. Watanabe et al, "Joint requirement”, EUV Source Workshop, Feb 2004, available at http://www.sematech.org 3 R.L. Kauffman, D.W. Phillion, and R.C. Spitzer, "X-ray production $\sim 13 \mathrm{~nm}$ from laser-produced plamsama for projection x-ray lithography applications," Appl. Opt. 32, 6897-6900 (1993) R. C. Spitzer, T.J. Orzechowski, D.W. Phillion, R.L. Kauffman, and C. Cerjan, "Conversion efficiencies from laser-produced-plasma in the extreme ultraviolet regime," J. Appl. Phys., 79, pp. 2251-2253 (1996

4 F. Jin, "Advanced laser plasma EUV source", a PhD thesis at Univ. of Central Florida (1995)

5 "New laser plasma source for extreme-ultraviolet lithography", F. Jin and M. Richardson, Applied Optics, Vol. 34, No. 25, pp. 5750-5760, September 1995.

6 "Water laser plasma x-ray point source and apparatus", by M. Richardson, M. Kado, K. Gabel \& F. Jin, Patent No. $5,459,771$, October 1995

7 D.A. Tichenor et al,"Diffraction-limited soft-x-ray projection imaging using a laser plasma source”, Opt. Lett. 16, 557 (1991)

8 "Cluster Beam Targets for Laser Plasma Extreme Ultraviolet and Soft X-ray Sources", Glenn D. Kublak and Martin Richardson, Patent No. 5,577,092, November 1996.

$9 \mathrm{~W}$. Svendsen and G. O'Sullivan, "Statistics and characteristics of xuv transition arrays from laser-produced plasmas of the elements tin through iodine," Phys. Rev. A, 50, 3710-3718 (1994)

G. M. Zeng, H. Daido, K. Murai, M. Nakatsuka, and S. Nakai, "Line x-ray emissions from highly ionized plasmas of various species irradiated by a compact solid-state lasers," J. Appl. Phys. 72, 3355-3362 (1992) 
10 B.A.M. Hansson, L. Rymel, M. Berglund, and H.M. Hertz, "A liquid-xenon-jet laser-plasma X-ray and EUV source”, Microelectronic Eng. 53, 667-670 (2000)

$11 \mathrm{H}$. Shields et al., "Xenon target performance characteristics for laser-produced plasma EUV sources," Proc. SPIE, vol. 4688, 94 (2002)

12 M. Richardson et al, "High conversion efficiency mass-limited-Sn-based laser-plasma source for EUV Lithography", Proc. 46th Int. Conf. on EIPBN (2003)

13 R. Benattar, C. Popovics, R. Sigel: Rev. Sci. Instr. 50 (12), 1583 (1979)

14 U. Kogelschatz: Appl. Opt. 13(8), 1749 (1974)

15 I. Hutchinson: Principles of Plasma Diagnostics, (Cambridge University Press, 1992) pp. 112-115

16 http://optics.tu-graz.ac.at/

17 J. Tawney, M. Richardson, R. Adhav: To be published

18 M. Kroon, R. Stuik, "Luminescent Materials for EUV Detection Purposes", SPIE, Vol. 4343, 665, (2001)

19 S.A. vd Westen et al, "Flying Circus 2: Calibration of an Extreme Ultraviolet Source at PLEX LLC," International SEMATECH Technology Transfer \#04024490A-TR (2004), available at http://www.sematech.org

20 N. Ceglio, A. Hawryluk, and R. Price, Lawrence Livermore Laboratory, report UCRL-86471, 1982. (unpublished) M. Schattenburg, H. Anderson, and H. Smith, Phys. Scr. 41, 13 (1990).

J. Weaver et al., Rev. Sci. Instrum. 72, 108 (2001).

21 M. Schattenburg, H. Anderson, and H. Smith, Phys. Scr. 41, 13 (1990).

22 J. Weaver et al., Rev. Sci. Instrum. 72, 108 (2001).

23 T. Kita, T. Harada, N. Nakano, and H. Kuroda, Appl. Opt. 22, 512 (1983)

24 W. Schwanda, K. Eidmann, and M. Richardson, J. X-Ray Sci Tech. 4, 1 (1993).

25 X-ray CCD camera model: PI-SX:512 from Princeton Instrument

26 International Radiation Detection (California, USA)

27 determined from tables at the CXRO website, http://www.cxro.lbnl.gov

28 K.Takenoshita et al, "Debris studies for the tin-based droplet laser-plasma EUV source," Proc. SPIE, vol 5374, pp. 954-963 (2004) 